\title{
Second-order relational properties and the inversion effect: Testing a theory of face perception
}

\author{
JAMES W. TANAKA and MARTHA J. FARAH \\ Carnegie-Mellon University, Pittsburgh, Pennsylvania
}

\begin{abstract}
Recognition of faces is more severely impaired by inversion than is recognition of other types of objects. This was originally interpreted as evidence for the existence of special face-recognition mechanisms. Recently, Diamond and Carey (1986) attributed the inversion effect to the use of second-order relational properties that are important for, but not unique to, face recognition. According to their hypothesis, face recognition differs from the recognition of most other objects in its dependence on second-order relational properties. This hypothesis was tested in two experiments by comparing the effects of inversion on the identification of dot patterns that differed in the extent to which they required the encoding of second-order relational properties. Identification of the second-order relational patterns was not more disrupted by inversion than was identification of first-order patterns. These results fail to support the hypothesis that second-order relational properties are responsible for the inversion effect.
\end{abstract}

The idea that face perception is somehow "special" has been suggested by work in developmental psychology showing the existence of innate templates for the human face (e.g., Morton \& Johnson, 1991), by clinical studies showing that face recognition ability can be selectively impaired by brain damage (e.g., DeRenzi, 1986), and by experimental studies with normal adults showing that face recognition is disrupted by inversion to a far greater extent than is the recognition of other types of objects. In this article, we will focus on the face inversion effect and test a recent and influential explanation of this phenomenon.

In the first demonstration of the face inversion effect, Yin (1969) presented subjects with upright or inverted pictures of faces, houses, airplanes, and other stimuli. After studying these pictures, subjects were then tested with stimuli in the same orientation in a forced-choice recognition paradigm. Yin found that when the stimuli were inspected and tested in the upright orientation, photographs of faces were better recognized than were photographs of other classes of objects. However, when the same stimuli were presented for inspection and test in the inverted orientation, recognition for faces declined below the recognition levels for the other object classes. The finding that upside-down faces appear to be disproportionately more difficult to recognize than other inverted objects has been referred to as the face inversion effect.

Subsequent studies have demonstrated the robust nature of the face inversion effect (see Valentine, 1988, for a review). Effects of inversion have been shown for famous

This research was supported by ONR Contract N0014-89-J3016, NIH Grants NS23458 and NS06209, NIH RCDA K04N501405, and NIMH Training Grant T32-MN-19102-02. Correspondence should be addressed to James W. Tanaka, Department of Psychology, Severance Lab, Oberlin, OH 44074 and novel faces (Scapinello \& Yarmey, 1970; Yarmey, 1971) and for simple line-drawn faces (Yin, 1969, Experiment 3). In other studies, inversion effects have been produced in which the stimulus materials are presented in blocks according to object class (Valentine \& Bruce, 1986) and in experiments in which the stimulus materials are randomly presented in a mixed list (Carey \& Diamond, 1977; Diamond \& Carey, 1986; Yin, 1969). Effects have also been obtained in experiments in which similar and dissimilar inspection-test orientations were used (Yin, 1969, Experiment 2). Recognition of faces also seems to be differentially impaired under conditions of inversion in studies requiring "old face" versus "new face" judgments (Scapinello \& Yarmey, 1970; Valentine \& Bruce, 1986; Yarmey, 1971). Thus, impaired recognition for inverted faces seems to be a robust and general phenomenon that cannot be explained simply as an artifact of stimulus material, experimental procedure, or task demands. It has therefore been interpreted as evidence for specialized facerecognition processes, which differ from the recognition processes used for other types of stimuli because they are keenly orientation-sensitive (e.g., Yin, 1969, 1970).

Ellis (1975) called into question the inference that disproportionate effects of inversion imply that qualitatively different recognition processes are being used. More recently, Diamond and Carey (1986) offered a specific alternative account of the face inversion effect, according to which the inversion effect is not specific to faces and therefore does not reflect processing that is unique to faces. Instead, Diamond and Carey suggested that the inversion effect results from the processing of a certain kind of spatial information that is particularly crucial for recognizing faces but is not necessarily unique to faces. They make a distinction between what they term first-order and 
second-order relational properties and attribute the inversion effect to the use of second-order relational properties in face recognition. First-order relational properties refer to the spatial relationships among parts of a stimulus. For example, the fact that the eyes are above the nose, or that a tree is to the left of the lake, are first-order relational descriptions. Second-order relational properties refer to the spatial configuration between the parts of a stimulus on the one hand and the central tendency or prototypical spatial configuration of its parts on the other. For a face with widely spaced eyes, the deviation of eye location on that face compared with the average face is an example of a second-order spatial relation. Of course, many stimuli that can be encoded with respect to their first-order relational properties cannot be encoded with respect to their second-order spatial relations because a prototypical configuration of their parts does not exist. Landscapes are an example of such a class of stimuli. There is no central tendency to the arrangement of trees, hills, and lakes in landscapes. Diamond and Carey also point out that even if a central tendency does exist for the arrangement of parts of a class of stimuli, the observer may not be able to represent these stimuli with respect to their second-order relational properties. To do so requires that the observer be equipped with knowledge of the prototypical configuration of the stimulus parts so that the second-order relations can be detected. Diamond and Carey suggested that of the stimuli whose spatial structures would allow for the encoding of second-order relational properties, the only class of stimuli for which most people have sufficient expertise to use second-order properties is faces. However, the use of second-order relational properties, and the consequent inversion effect, is not necessarily limited to faces. In support of this view, Diamond and Carey showed that inversion effects are obtained with faces for all subjects and with dogs for dog experts.

Diamond and Carey's (1986) account of the face inversion effect has been widely influential, if recent discussions of the inversion effect in textbooks (e.g., Humphreys \& Bruce, 1989) and monographs and review papers on face perception (e.g., Bruce, 1988; Valentine, 1988; Young, 1988) are any indication. However, there are two separate claims in Diamond and Carey's hypothesis that may not have been adequately distinguished. First, Diamond and Carey make a claim about the nonuniqueness of the inversion effect to face processing. They argue that the inversion effect does not index a perceptual process unique to face perception but rather a perceptual process that is used for identifying highly similar stimuli by viewers who are expert in the stimulus domain. They support this claim with their experiment on dog recognition by dog experts. Second, they make a claim about the reason for the inversion effect. This part of their hypothesis is of great interest because it provides an account of "expert" recognition (face perception for normal peo- ple and dog perception for dog experts) that explains how it differs from other forms of object recognition. They argue that expert recognition is qualitatively different from nonexpert recognition because it relies on second-order relations. However, this part of the hypothesis has no direct support. There is no a priori reason to expect that second-order relational properties are more sensitive to inversions than are first-order relational properties. Nothing in the concept of a prototypical configuration, or the assessment of deviations from a prototype, implies orientation-specificity. In addition, there is no direct empirical support for this conjecture, in the sense of a controlled experiment in which first-order versus secondorder relational properties were directly manipulated and the effect of this manipulation on recognition of misoriented stimuli measured. Although Diamond and Carey predicted on the basis of their hypothesis that dog experts would show an inversion effect for dogs, they used complex realistic stimuli and different subject populations that varied from one another in many ways, and it is possible that the critical variable for producing the inversion effect was not the use of second-order relational properties. For example, Tanaka and Taylor (1991) have found that dog and bird experts differ from nonexperts in the sheer number of properties they use to identify animals in their areas of expertise. Therefore, as simple, elegant, and eminently testable as the second-order relational hypothesis is, it has not been directly tested. The goal of the following two experiments is to test this hypothesis.

\section{EXPERIMENT 1}

In this experiment, the subjects were taught to identify dot patterns that either shared or did not share a spatial configuration. Dot patterns were selected as the stimulus material because they are discriminable solely on the basis of relational information. Dot patterns that do not share a configuration are discriminable solely on the basis of first-order relational information and will therefore be referred to as "first-order patterns." As pointed out by Diamond and Carey (1986), patterns that do share a configuration can be discriminated on the basis of both firstorder and second-order relations. However, because these patterns will have highly similar first-order relations, subjects' identifications of them will be aided by the encoding of second-order relational information. In addition, the classic research of Posner and Keele (1968) shows that subjects spontaneously abstract the shared configuration of groups of dot patterns such as those used in this experiment, suggesting that second-order relations in these stimuli will, in fact, be available to subjects. For this reason, the dot patterns that share a configuration will be referred to as "second-order patterns." To test the hypothesis that second-order relations are more sensitive to inversion than are first-order relations, it is not necessary that subjects encode the second-order patterns solely in 
terms of their second-order relations; it is only necessary that subjects use relatively more second-order relational information in identifying the second-order patterns.

In the training phase of this experiment, the subjects learned to identify the first-order and second-order patterns by female names or male names. In the test phase, the subjects were asked to identify the same dot patterns presented in their upright and inverted orientations. Based on Diamond and Carey's (1986) hypothesis, the identification of the second-order patterns should be more impaired when presented in the inverted orientation than should the first-order patterns.

\section{Method}

\section{Subjects}

Twenty-eight undergraduate psychology students from CarnegieMellon University participated in the experiment. The subjects were tested individually and received course credit for their participation.

\section{Materials}

The experimental stimuli were a series of dot patterns generated first by assigning nine dots to randomly determined coordinate locations on a $40 \times 30$ grid. Seven basic template patterns were constructed in this manner. To obtain a set of patterns that shared the same configural shape, the template patterns were probabilistically distorted (Posner \& Keele, 1968). A $5 \times 5$ matrix was defined around each dot in the template pattern. Excluding the original location of the dot on the template pattern, there were 24 new locations to which the dot could be relocated. A probabilistic rule was then applied that randomly selected one of the 24 possible locations for the new position of the dot on the second-order pattern. All locations had an equal probability of selection. The second-order pattern was created by applying the probabilistic selection rule to all nine dots on the template pattern. Six second-order patterns for each of the 7 template patterns were generated. Thus, in the stimulus set, there were a total of $\mathbf{4 2}$ patterns (only the second-order patterns were used in the experiment). In the second-order pattern condition, the 6 patterns originated from the same template. In the
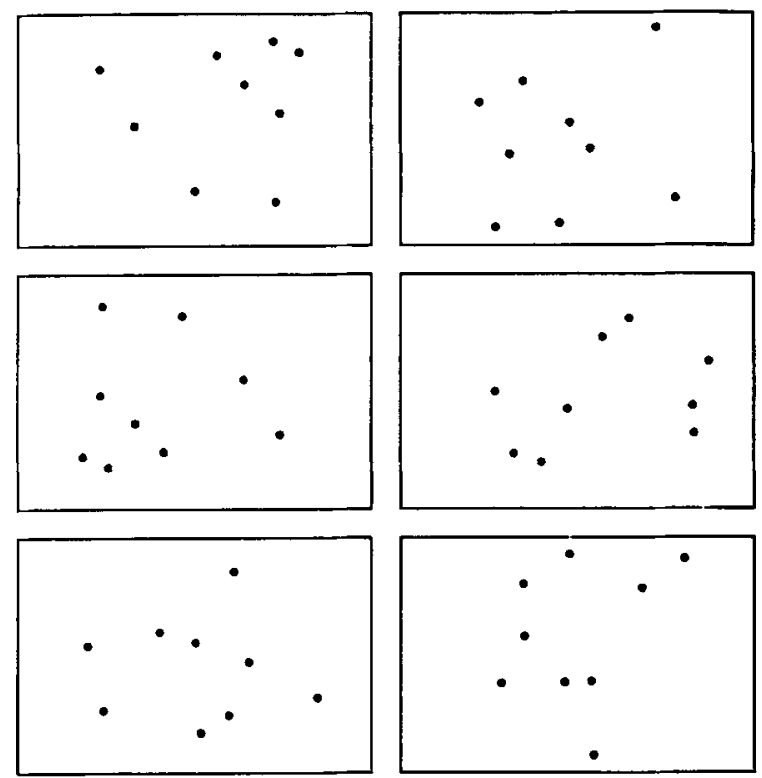

Figure 1. Examples of the first-order relational patterns used in Experiment 1.
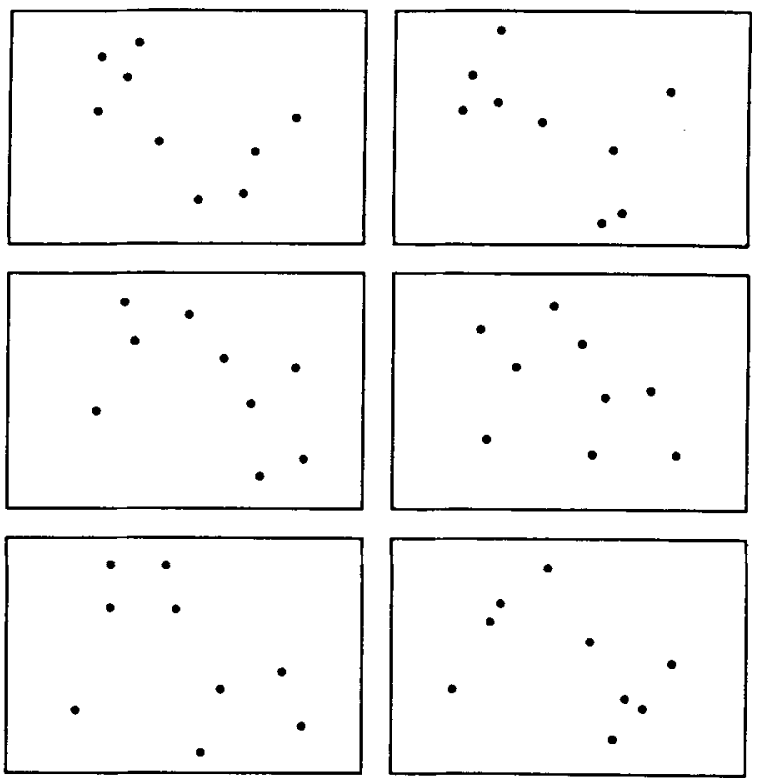

Figure 2. Examples of the second-order relational patterns used in Experiment 1.

first-order pattern condition, the patterns were derived from the other 6 template patterns. For example, in the second-order pattern condition, a subject may see the 6 patterns derived from Template Pattern A. In the first-order pattern condition, the same subject would see one pattern derived from each of the remaining 6 template patterns (i.e., B, C, D, E, F, G). Examples of first-order and second-order patterns are shown in Figures 1 and 2 . Each pattern appeared an equal number of times as a member of the secondorder and first-order pattern sets.

The patterns were reduced, photocopied, and pasted onto $7 \times$ 6 in. white card stock. To discourage subjects from using idiosyncratic surface information as a means of identification, six copies of each pattern were made: two copies for the training set and four copies for the test set (two for presentation in the upright orientation and two for presentation in the inverted condition).

\section{Procedure}

The subjects were seated at a table and directly faced the experimenter at a viewing distance of approximately $2 \mathrm{~m}$. The subjects were informed that they would see six dot patterns and that their task would be to learn to associate names with the patterns. In the second-order condition, the six patterns were generated from the same template pattern. In the first-order condition, the six patterns were generated from different template patterns.

Learning phase. During the learning phase of the experiment, the subjects were shown six to-be-learned (second-order or firstorder) dot patterns for inspection. After initial inspection, the experimenter introduced the individual dot patterns by their assigned names. Patterns in each condition were given either female names (Sue, Betty, Joan, Mary, Ruth, Ann, Kate) or male names (Joe, Bob, Sam, Tom, Bill, Dave, Ted). Each pattern was introduced twice. The experimenter randomly presented each stimulus pattern twice, with the restriction that the same pattern could not be presented on consecutive trials. The subject's task was to identify the pattern by its name. If the subject responded incorrectly, the experimenter provided the correct name and recorded the incorrect response. Training continued until the subject was able to correctly identify the six patterns twice without error.

Test phase. Immediately following training, a recall test was administered in which the subject was asked to identify the pattern 
randomly presented in either its upright or inverted orientation. The subjects' responses were recorded by the experimenter, and no feedback was provided. Each dot pattern was presented four times, twice in the upright orientation and twice in the inverted orientation, with upright and inverted orientations randomly intermixed and with the restriction that the same pattern could not be presented on consecutive trials. If the subjects were not certain of the identity of the pattern, they were told to guess. After testing, the same procedure was repeated with patterns from the untested pattern-type condition. Presentation order of the pattern type (second-order and firstorder) and assignment of gender names to pattern type were counterbalanced across subjects.

\section{Results and Discussion}

\section{Training Trials}

Means of 2.75 and 4.53 training runs were required for the subjects to learn the first- and second-order patterns, respectively. The difference in means was reliable by a matched-pairs Wilcoxon test $[T(28)=83, p<.05]$. The higher number of training runs needed to learn the second-order patterns is not surprising, given the patterns' greater similarity to one another. As Diamond and Carey (1986) have pointed out, second-order relations will only be encoded if the patterns are so similar that first-order relations alone are not sufficient to distinguish among them. Thus, the difference in similarity between the two groups of patterns does not constitute a confound but is an integral part of the hypothesis being tested. Because the subjects were trained to the same criterion for recognition of the upright patterns from both groups, the difference in similarity does not lead to a difference in difficulty for the identification trials. The greater number of training runs required for the subjects to learn the second-order patterns is consistent with Diamond and Carey's claim that discriminations based on second-order relational properties demand a type of perceptual expertise that is not required for first-order discriminations.

\section{Identification Errors}

The critical test of the hypothesis that the use of secondorder relational properties underlies the inversion effect was whether inversion caused a more severe performance decrement in second-order than in first-order pattern identification. The means shown in Figure 3 suggest that this is not the case. In the upright orientation, $93 \%$ of the second-order patterns were correctly identified, compared with $87 \%$ in the inverted orientation. Similarly, $94 \%$ of the first-order patterns were correctly identified in the upright presentations, compared with $88 \%$ in the inverted presentations. For both types of patterns, inversion resulted in a decrement of $6 \%$. There was a reliable effect of orientation in the expected direction $[T(28)=52$, $p<.01]$ but no effect of pattern type $[T(28)=161$, $p>.10]$.

Could the absence of the predicted interaction be attributed to a ceiling or floor effect? Performance in both conditions is well above chance, which rules out a floor effect. A ceiling effect obscuring different levels of competence with upright first- and second-order patterns is unlikely, given our training procedure. Training with each

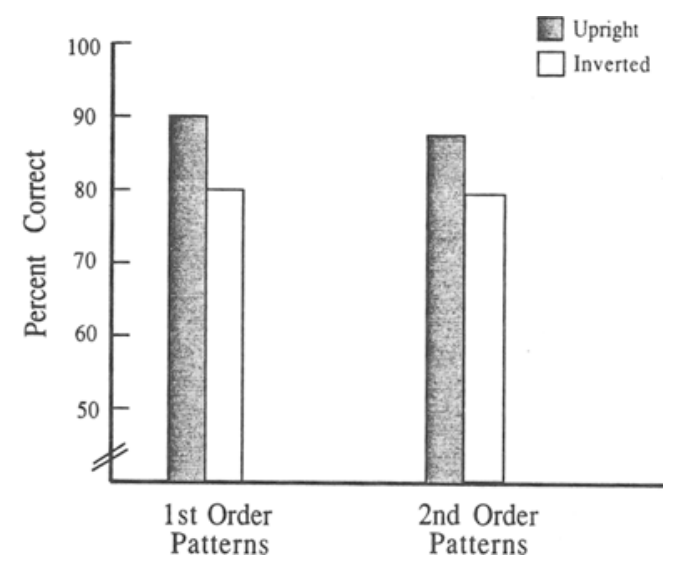

Figure 3. Percentage of correctly identified first-order and secondorder relational patterns presented in their upright and inverted orientations in Experiment 1.

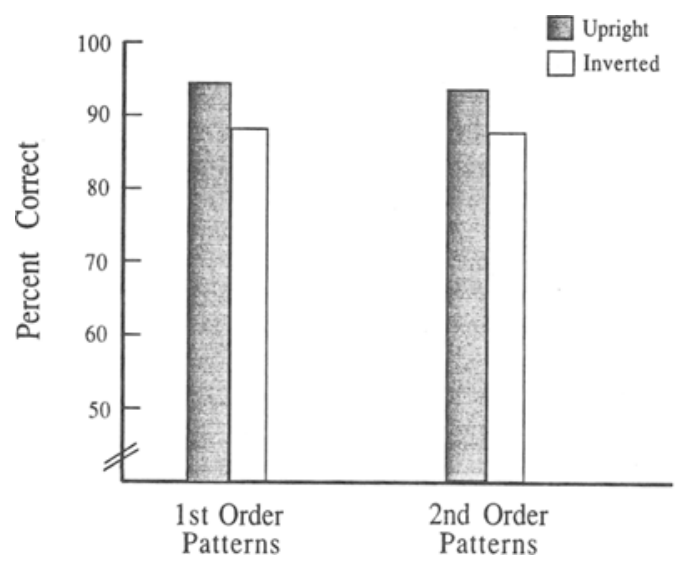

Figure 4. Identification performance of subjects committing four or more errors for first-order and second-order relational patterns presented in their upright and inverted orientations in Experiment 1.

pattern type was terminated as soon as the subjects reached equivalent levels of performance. However, to eliminate this possibility, we carried out an additional analysis of the data from this experiment. A second test was performed on the 15 subjects who made at least four identification errors. The average performance of this subgroup was, predictably, lower than that of the entire group of subjects. Nevertheless, as shown in Figure 4, the same pattern of results was obtained: first-order patterns were correctly identified on $90 \%$ of the trials in their upright orientation and on $80 \%$ of the trials in their inverted orientation; second-order patterns were correctly identified on $88 \%$ of the trials in their upright orientation, and on $79 \%$ of the trials in their inverted orientation.

Is it possible that the subjects failed to notice the shared configuration of the patterns in the second-order relations condition and therefore treated them like the first-order patterns? Most subjects spontaneously commented that the patterns in the second-order group shared a configuration. 
However, it is still possible that they mainly used firstorder relations in learning them. Therefore, in Experiment 2, to maximize the relevant difference between the two groups of patterns, we increased the degree to which patterns in the second-order group shared a configuration.

\section{EXPERIMENT 2}

This experiment is similar in design to Experiment 1, with one exception. The patterns in the second-order relations condition of this experiment shared a higher degree of configuration, increasing the likelihood of the subjects' encoding second-order relations to distinguish among these patterns.

\section{Method}

\section{Subjects}

Twenty-eight undergraduate psychology students from CarnegieMellon University served as subjects in the experiment. The subjects were tested individually and received course credit for their participation.

\section{Materials}

The template patterns from Experiment 1 were used as the template patterns in the current experiment. To increase the amount of shared configuration, the $5 \times 5$ matrix that was used to distort second-order patterns in Experiment 1 was reduced to a $3 \times 3$ matrix. Excluding the original location of the dot on the template pattern, there were eight new locations on the second-order pattern to which a dot could be relocated. A probabilistic rule selected one of the eight possible locations for the new position of the dot. All locations had an equal probability of selection. The second-order pattern was created by applying the probabilistic selection rule to all nine dots on the template pattern. Once again, 6 second-order patterns for each of the 7 template patterns were generated, for a total of 42 patterns. An example of one second-order pattern set used in Experiment 2 is shown in Figure 5.
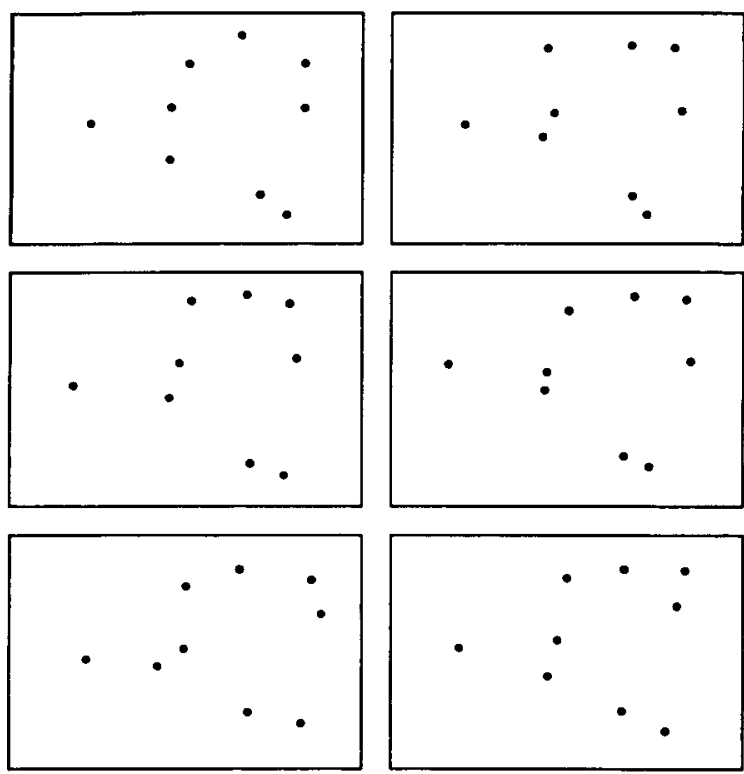

Figure 5. Examples of the second-order relational patterns used in Experiment 2.

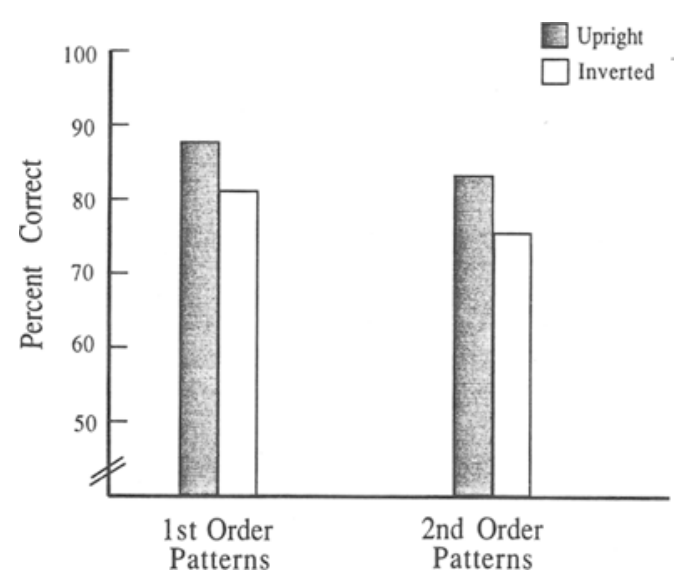

Figure 6. Percentage of correctly identified first-order and secondorder relational patterns presented in their upright and inverted orientations in Experiment 2.

\section{Procedure}

The same procedure described in Experiment 1 was used. Training continued until a learning criterion of one error or no errors per run was reached. After engaging in a 5-min filler task, the subjects were given a recall test in which they identified four presentations of the six patterns, which were presented twice in their upright orientation and twice in their inverted orientation. The orientation of the pattern was randomly intermixed across presentation trials, with the restriction that the same pattern could not be presented (either upright or inverted) on consecutive trials. After patterns from one condition were tested, the procedure was repeated with patterns from the untested condition. As before, order of the initial pattern type (first-order and second-order) and assignment of gender names to pattern type were counterbalanced across subjects.

\section{Results and Discussion}

\section{Training}

In the training phase, means of 2.39 and 4.71 training runs were required for the subjects to learn the first- and second-order patterns, respectively. The difference was reliable $[T(28)=56, p<.01]$ and again demonstrates that learning second-order relational properties requires more experience than learning first-order relational properties.

\section{Identification Errors}

Figure 6 shows the mean performance in the four conditions that are critical for testing the second-order relations account of the inversion effect. As in the previous experiment, the effects of inversion were not reliably greater for the second-order than for the first-order patterns. First-order relational patterns were correctly identified on $88 \%$ and $81 \%$ of the trials in the upright and inverted orientations, respectively. Second-order relational patterns were correctly identified on $83 \%$ and $75 \%$ of the trials in the upright and inverted orientations, respectively. Thus, inversion resulted in $7 \%$ and $8 \%$ decrements for the first- and second-order patterns, respectively. This difference was not reliable by the Wil- 
coxon test $[T(28)=172, p>.10]$. As before, orientation affected identification $[T(28)=32, p<.01]$ but not pattern type $[T(28)=123, p>.10]$.

\section{GENERAL DISCUSSION}

Although inversion will make most stimuli somewhat harder to recognize, certain stimuli become dramatically less recognizable when inverted. Faces are the most common example of such stimuli, but there are other examples as well, as Diamond and Carey (1986) have demonstrated with their experiment on dog recognition by dog experts. Why is inversion so disruptive to perception in these cases? What is the nature of dog perception by dog experts, and face perception by all of us, that is so vulnerable to a change in stimulus orientation?

Diamond and Carey (1986) proposed a simple and elegant answer to this question, which has been widely accepted. They suggested that the inversion effect results from the use of second-order relational properties. However, their research on the inversion effect in face perception and in dog perception never included a direct test of this important claim.

We tested Diamond and Carey's (1986) explanation of the inversion effect by comparing the size of inversion effect obtained when subjects recognized dot patterns that were either distinguishable on the basis of first-order relational properties or shared a spatial configuration and were thus most easily discriminable on the basis of second-order relational properties. In two studies, inversion was found to have comparable effects on the two kinds of dot patterns. This suggests that, contrary to the hypothesis of Diamond and Carey, second-order relational properties are no more vulnerable to inversion than are first-order relational properties.

Two notes of caution should be added to these conclusions. First, our stimuli were very different from faces, and the expertise of our subjects for these stimuli was much less than the expertise of normal people for faces or dog-show judges for dogs. Thus, the second-order relational properties hypothesis was tested by us under conditions very different from face and natural-object recognition. However, to test the claim that the inversion effect in face perception is caused by the use of second-order relational properties, it is not only permissible, but desirable, to use stimuli that vary only in the availability of second-order relational properties and that are different from faces in their visual properties and familiarity.

Second, our finding is a null result of sorts, and caution is therefore required in interpreting it. Nevertheless, several considerations support our argument that our experiments had sufficient sensitivity. In both experiments, there was evidence of reliable differences both in the way in which the subjects learned the first- and second-order relational patterns and between the subjects' recognition of upright and inverted patterns. In previous studies of inversion effects with face stimuli, the difference between the effects of inversion on the recognition faces and other objects is at least as great as the effects of inversion on nonface objects (e.g., Diamond \& Carey, 1986, Experiment 1; Yin, 1969). Therefore, given that our paradigm showed highly reliable inversion effects for the dot patterns in general, it should have been capable of detecting the predicted differential effects of inversion on the firstand second-order relational patterns.

Do the present results conflict with those of Diamond and Carey (1986)? No, they merely suggest that it was not the use of second-order relational properties that led to an inversion effect for their dog experts. Taking our results together with theirs, the implications for face perception are the following: There is indeed a "special" orientation-sensitive process used in face perception, as well as in the perception of certain other highly similar stimuli when expertise is sufficiently high. However, the nature of that process is currently unknown. This is a fundamental question about face perception and perceptual expertise that awaits further research.

\section{REFERENCES}

Bruce, V. (1988). Recognizing faces. London: Erlbaum.

Carey, S., Diamond, R. (1977). From piecemeal to configurational representation of faces. Science, 195, 312-314.

DeRenzI, E. (1986). Current issues in prosopagnosia. In H. D. Ellis, M. A. Jeeves, F. Newcombe, \& A. Young (Eds.), Aspects of face processing (pp. 243-251). Dordrecht, The Netherlands: Martinus Nijhoff.

Diamond, R., Carey, S. (1986). Why faces are and are not special: An effect of expertise. Journal of Experimental Psychology: General, 115, 107-117.

ELus, H. D. (1975). Recognizing faces. British Joumal of Psychology, 66, 409-426.

Humphreys, G. W., Bruce, V. (1989). Visual cognition: Computational, experimental, and neuropsychological perspectives. Hillsdale, NJ: Erlbaum.

Morton, J., Johnson, M. H. (1991). CONSPEC and CONLERN: A two-process theory of infant face recognition. Psychological Review, 98, 164-181.

Posner, M. I., \& KeELe, S. W. (1968). On the genesis of abstract ideas. Joumal of Experimental Psychology, 77, 353-363.

Scapinello, K. F., Y YRMeY, A. D. (1970). The role of familiarity and orientation in immediate and delayed recognition of pictorial stimuli. Psychonomic Science, 21, 329-331.

TANAKA, J. W., TAYloR, M. (1991). Object categories and expertise: Is the basic level in the eye of the beholder? Cognitive Psychology, 23, 457-483.

VAlentine, T. (1988). Upside-down faces: A review of the effect of inversion upon face recognition. British Journal of Psychology, 79, $471-491$.

VALENTINe, T., BRuce, V. (1986). The effect of race, inversion and encoding activity upon face recognition. Acta Psychologica, 61, 259-273.

Y ARMEY, A. D. (1971). Recognition memory for familiar "public" faces: Effects of orientation and delay. Psychonomic Science, 24, 286-288.

YIN, R. K. (1969). Looking at upside-down faces. Joumal of Experimental Psychology, 81, 141-145.

YIN, R. K. (1970). Face recognition by brain-injured patients: A dissociable ability? Neuropsychologia, 8, 395-402.

YouNG, A. (1988). Functional organization of visual recognition. In L. Weiskrantz (Ed.), Thought without language (pp. 78-107). Oxford: Oxford University Press.

(Manuscript received December 13, 1990; revision accepted for publication June 24,1991 .) 